\title{
EIGENVALUES AND EIGENFUNCTIONS OF RIEMANNIAN MANIFOLDS ${ }^{1}$
}

\author{
FRIEDER-JENS LANGE AND UDO SIMON
}

\begin{abstract}
S.-Y. Cheng [Proc. Amer. Soc. 55 (1976), 379-381] investigated closed two-dimensional Riemannian manifolds of genus zero which admit $\boldsymbol{m}$ first eigenfunctions with constant square sum, $m>1$. In this note, we will investigate $n$-dimensional Riemannian manifolds with $m$ eigenfunctions, corresponding to the eigenvalue $\lambda$, and with constant square sum. Examples of such manifolds are minimal submanifolds of spheres. While Cheng investigated closed manifolds, most of our results have local character. We give lower bounds for $\lambda$ by curvature functions (scalar curvature, sectional curvature) and apply these results in two cases: (i) to characterize manifolds which are locally isometric to spheres; (ii) to the investigation of minimal submanifolds of spheres. These results extend earlier results of Lange and Simon.
\end{abstract}

1. Notations and auxiliary results. Let $(M, g)$ be a connected Riemannian manifold of class $C^{\infty}, n=\operatorname{dim} M>2$; denote by $\nabla$ the corresponding covariant differentiation and by $g_{i j}$ (resp., $g^{i j}$ ) the components of the metric tensor $g$ (resp., $g^{-1}$ ) in local coordinates $\left(u^{i}\right)$; denote by $R_{i j k}^{h}$ (resp., $R_{i j}$ ) the components of the curvature tensor (resp., the Ricci tensor) (with the sign of [5, p. 201]); let $R$ denote the scalar curvature (such that $R=1$ on the unit sphere) and $\kappa$ the sectional curvature. As usual, raising and lowering of indices are defined. Let $f: M \rightarrow \mathbf{R}$ be a $C^{\infty}$-function, let $f_{i j}:=\nabla_{i} \nabla_{j} f$ denote the components of the Hessian $\operatorname{Hess}(f)$ and denote the Laplacian by $\Delta f:=g^{i j} f_{i j}$.

(1.1) LemMa [7]. Let $f: M \rightarrow \mathbf{R}$ be a $C^{\infty}$-function. Then $f$ satisfies

$$
\begin{aligned}
\frac{1}{2} \Delta\left(f_{i j} f^{i j}\right)= & 2 \sum_{i<j} \kappa_{i j}\left(\sigma_{i}-\sigma_{j}\right)^{2}+f^{i j} \nabla_{j} \nabla_{i}(\Delta f) \\
& +\nabla_{k} f_{i j} \nabla^{k} f^{i j}+f^{i j} f^{h}\left[2 \nabla_{i} R_{j h}-\nabla_{h} R_{i j}\right]
\end{aligned}
$$

where $\sigma_{1}, \ldots, \sigma_{n}$ are the eigenvalues of the Hessian with corresponding orthonormal eigenvectors $E_{1}, \ldots, E_{n}$ and $\kappa_{i j}$ is the sectional curvature of the plane $\left\{E_{i}, E_{j}\right\}_{i \neq j}$

Received by the editors January 18, 1978 and, in revised form, June 29, 1978.

1980 mathematics subject classification. Primary 35P15, 53C25, 58G25; Secondary 53B20, 53B25, 53C20, 53C40.

Key words and phrases. Lower bounds of eigenvalues, curvature, isometries with spheres, minimal submanifolds of spheres.

${ }^{1}$ The authors thank D. Koutroufiotis and H. Wissner for valuable hints. 
2. Eigenfunctions. In the following we will investigate Riemannian manifolds of dimension $n>2$. Let $f(\alpha): M \rightarrow \mathbf{R}, 1<\alpha<m$, be eigenfunctions corresponding to $\lambda \neq 0$, i.e., $\Delta f(\alpha)+\lambda f(\alpha)=0$; additionally we assume that for an $m \in \mathbf{N}, m>1$ :

$$
\sum_{\alpha=1}^{m} f^{2}(\alpha)=\text { const }=c>0 .
$$

Examples of such manifolds are minimal submanifolds of spheres, which we study in the last section.

We make the following calculations: From the differential equation for $f$ and (2.1) we get

$$
0=\sum_{\alpha} f(\alpha) f(\alpha)_{i}
$$

and

$$
0=\Delta \sum_{\alpha} f^{2}(\alpha)=2 \sum_{\alpha}\|\operatorname{grad} f(\alpha)\|^{2}+2 \sum_{\alpha} f(\alpha) \Delta f(\alpha)
$$

therefore

$$
\sum_{\alpha}\|\operatorname{grad} f(\alpha)\|^{2}=\lambda \cdot c \quad \text { and } \quad \sum_{\alpha} \nabla_{i}\left(f(\alpha)^{i} \Delta f(\alpha)\right)=0 .
$$

(2.1)-(2.3) and the Bochner-Lichnerowicz formula [1, p. 131]

$$
\frac{1}{2} \Delta\|\operatorname{grad} f\|^{2}=f_{i j} j^{i j}-(\Delta f)^{2}+R^{i j} f_{i} f_{j}+\nabla_{i}\left(f^{i} \Delta f\right)
$$

imply

$$
\sum_{\alpha} f(\alpha)_{i j} f(\alpha)^{i j}=\lambda^{2} c-\sum_{\alpha} R^{i j} f(\alpha)_{i} f(\alpha)_{j}
$$

If $\sigma(\alpha)_{1}, \ldots, \sigma(\alpha)_{n}$ are the eigenvalues of $\operatorname{Hess}(f(\alpha))$, then

$$
n f(\alpha)_{i j} f(\alpha)^{i j}=\sum_{i<j}\left(\sigma(\alpha)_{i}-\sigma(\alpha)_{j}\right)^{2}+(\Delta f(\alpha))^{2}
$$

(2.1) and (2.5) imply

$$
(n-1) \cdot \lambda^{2} c=n \cdot \sum_{\alpha} R^{i j} f(\alpha)_{i} f(\alpha)_{j}+\sum_{\alpha} \sum_{i<j}\left(\sigma(\alpha)_{i}-\sigma(\alpha)_{j}\right)^{2} .
$$

(2.7) LEMMA. If $(M, g)$ is a connected Riemannian manifold with $m$ eigenfunctions $f(1), \ldots, f(m)$ corresponding to the eigenvalue $\lambda, m>1$, and if (2.1) is satisfied, then $\lambda>0$ and furthermore

$$
\lambda^{2}>\frac{n}{(n-1) c} R^{i j} \sum_{\alpha} f(\alpha)_{j} f(\alpha)_{j} .
$$

Equality holds iff $f(\alpha)_{i j}+\lambda f(\alpha) \cdot g_{i j}=0$ for $\alpha=1, \ldots, m$.

Proof. Assume equality in (2.7); then (2.6) implies $\sigma(\alpha)_{1}=\sigma(\alpha)_{2}=\cdots=$ $\sigma(\alpha)_{n}=: \sigma(\alpha)$ for $\alpha=1, \ldots, m$, so $f(\alpha)_{i j}=\sigma(\alpha) \cdot g_{i j}$, which gives the assertion because $-\lambda f(\alpha)=\Delta f(\alpha)=n \cdot \sigma(\alpha)$. 
3. Einstein spaces. In this section we investigate Einstein spaces of dimension $n>3\left(R_{i j}=(n-1) R \cdot g_{i j}\right.$ with $R=$ const $)$, which admit eigenfunctions $f(\alpha), 1<\alpha<m$, such that (2.1) is satisfied. A simple consequence of (2.7) is the following lemma, which is an analogue to Theorem 1 in [6] and which gives a lower bound for the first positive eigenvalue $\lambda_{1}$.

(3.1) LEMMA. Let $(M, g)$ be a connected Einstein space with $m$ eigenfunctions corresponding to the eigenvalue $\lambda, m>1$, which satisfy (2.1). Then $\lambda>n R$ and equality holds iff $f(\alpha)_{i j}+\lambda f(\alpha) g_{i j}=0$ for $\alpha=1, \ldots, m$.

(3.2) Theorem. Let $(M, g)$ be a connected Einstein space, $n=\operatorname{dim} M>3$, with $m>1$ eigenfunctions $f(1), \ldots, f(m)$ corresponding to $\lambda$ which satisfy (2.1). Then either $\lambda_{1}=n R=n \kappa_{0}$, and $f(\alpha)_{i j}+(\lambda / n) f(\alpha) g_{i j}=0$ for $\alpha=1, \ldots, m$, or $\lambda_{1}>2 n \kappa_{0}$, where $\kappa_{0}=\inf \kappa$ on $M$.

(3.3) REMARK. Related results for closed Einstein spaces were proved in [9], [13]; (3.2) first was proved in [4].

Proof of (3.2). Each $f(\alpha)$ satisfies (1.3); therefore

$$
\begin{aligned}
\frac{1}{2} \Delta\left(\sum_{\alpha} f(\alpha)_{i j} f(\alpha)^{i j}\right)= & \sum_{\alpha} \sum_{i<j} 2 \kappa(\alpha)_{i j}\left(\sigma(\alpha)_{i}-\sigma(\alpha)_{j}\right)^{2} \\
& -\lambda \sum_{\alpha} f(\alpha)_{i j} f(\alpha)^{i j}+\sum_{\alpha} \nabla_{k} f(\alpha)_{i j} \nabla^{k} f(\alpha)^{i j}
\end{aligned}
$$

We make the following calculations:

(A) As $R_{i j}=(n-1) \cdot R \cdot g_{i j}$ (with $R=$ const for $n>3$ ), (2.3) and (2.5) imply

$$
\sum_{\alpha} f(\alpha)_{i j} f(\alpha)^{i j}=\lambda c(\lambda-(n-1) \cdot R) .
$$

(B) From (2.3) and (2.6) we have for an Einstein space

$$
\sum_{\alpha} \sum_{i<j}\left(\sigma(\alpha)_{i}-\sigma(\alpha)_{j}\right)^{2}=\lambda c(n-1)(\lambda-n R) .
$$

(C) Denote by $A(\alpha)_{i j}:=f(\alpha)_{i j}+(\lambda / n) \cdot f(\alpha) \cdot g_{i j}$; then

$$
0<\nabla_{k} A(\alpha)_{i j} \nabla^{k} A(\alpha)^{i j}=\nabla_{k} f(\alpha)_{i j} \nabla^{k} f(\alpha)^{i j}-\frac{\lambda^{2}}{n}\|\operatorname{grad} f(\alpha)\|^{2}
$$

Introducing (3.4b), (3.4c) and (3.4d) into (3.4a), we get

$$
0>n^{-1}(n-1) \lambda c(\lambda-n R)\left(2 n \kappa_{0}-\lambda\right)+\sum_{\alpha} \nabla_{k} A(\alpha)_{i j} \nabla^{k} A(\alpha)^{i j} .
$$

Assume $2 n \kappa_{0}>\lambda$; as $\lambda>n R$ from (3.1) and $\lambda c>0$ from (2.1) and (2.7), each summand in (3.4e) must vanish. $(M, g)$ is irreducible, as $\kappa_{0}>(2 n)^{-1} \cdot \lambda>0$, therefore

$$
\nabla_{k} A(\alpha)_{i j}=0 \quad \text { iff } f(\alpha)_{i j}+\frac{\lambda}{n} f(\alpha) g_{i j}=\mu(\alpha) g_{i j}, \quad \mu(\alpha) \in \mathbf{R} .
$$

Trace $A(\alpha)=0$ gives $\mu(\alpha)=0$, therefore (3.4f) implies $A(\alpha)=0$. But then 
$\sigma(\alpha)_{i}=\sigma(\alpha)_{j}$ for $i, j=1, \ldots, m$ and $\lambda-n R=0$ from (3.4c). This proves the assertion (Lemma 3.1).

4. Local isometries with spheres. The results of the preceding and the following sections together with results of $K$. Tandai [11] give local characterizations of spheres; as an example we formulate Corollary (4.1) below; it is obvious how to formulate further results.

(4.1) Corollary. Let $(M, g)$ be a connected Einstein space, $n=\operatorname{dim} M>$ 3 , with $(n+1)$ eigenfunctions $f(1), \ldots, f(n+1)$ corresponding to $\lambda>0$, linearly independent over $\mathbf{R}$, which satisfy (2.1). Then either $\lambda_{1}=n R$ and there exists an isometric immersion $\Phi: M \rightarrow S^{n}(1 / \sqrt{R})$ or $\lambda>2 n \kappa_{0}$.

5. Two-dimensional Riemannian manifolds. In [9, 83], we proved for a closed two-dimensional manifold, that each eigenvalue $\lambda \neq 0$ satisfies $\lambda>2$ $\min _{p \in M} \kappa(p)$ (which is of interest only if $\min \kappa>0$ ). As each two-dimensional Riemannian manifold is an Einstein space $\left(R_{i j}=\kappa \cdot g_{i j}\right)$, from (2.7) we get the following result (cf. [2, p. 380]). (Note that (3.4a)-(3.4d) are valid for two-dimensional manifolds too, where $R=\kappa$ generally is not a constant.)

(5.1) Lemma. Let $(M, g)$ be a connected two-dimensional Riemannian manifold. If $M$ admits $m$ eigenfunctions, $m>1$, corresponding to the eigenvalue $\lambda$, which satisfy (2.1), then $\lambda>2$ sup $\kappa$, and equality holds iff $(M, g)$ is locally isometric to a sphere and $\lambda$ is the first eigenvalue.

Proof. Equality in (5.1) implies that there exist $m(m>2)$ functions which satisfy the system $f(\alpha)_{i j}+\lambda f(\alpha) g_{i j}=0$; apply Tandai's results [11].

(5.2) Lemma. Let $(M, g)$ be a connected Riemannian manifold $\operatorname{dim} M=2$, with $m$ eigenfunctions $f(1), \ldots, f(m)$ corresponding to the eigenvalue $\lambda, m>1$, which satisfy (2.1). Then the curvature $\kappa$ of $(M, g)$ fulfils the differential inequality $\Delta \kappa<(\lambda-2 \kappa)(\lambda-4 \kappa)$.

Proof. (2.5) implies $\sum_{\alpha=1}^{m} f(\alpha)_{i j} f(\alpha)^{i j}=\lambda c(\lambda-\kappa)$; from (1.1), (3.4b) and $R_{i j}=\kappa \cdot g_{i j}$ we get

$$
\begin{aligned}
0= & \frac{1}{2} \lambda c \cdot \Delta \kappa+\sum_{\alpha} 2 \kappa\left(\sigma(\alpha)_{1}-\sigma(\alpha)_{2}\right)^{2}-\lambda^{2} c(\lambda-\kappa) \\
& +\sum_{\alpha} \nabla_{k} f(\alpha)_{i j} \nabla^{k} f(\alpha)^{i j}+\sum_{\alpha} f(\alpha)^{i j} f(\alpha)^{h}\left[2 \kappa_{i} g_{j h}-\kappa_{h} g_{i j}\right] .
\end{aligned}
$$

Using (3.4c) and (3.4d) for $n=2, R=\kappa,(5.2 .1)$ gives

$$
\begin{aligned}
0= & \frac{1}{2} \lambda c[\Delta \kappa-(\lambda-4 \kappa)(\lambda-2 \kappa)]+\left\{\sum_{\alpha} \nabla_{k} f(\alpha)_{i j} \nabla^{k} f(\alpha)^{i j}-\frac{1}{2} \lambda^{3} c\right\} \\
& +2 \kappa_{i} \sum_{\alpha} f(\alpha)^{i j} f(\alpha)_{j}-\kappa_{h} \sum_{\alpha} \Delta f(\alpha) \cdot f(\alpha)^{h} .
\end{aligned}
$$

The last two terms vanish because of (2.2) and (2.3); as $\{\cdots\}>0$ from (3.4d) and (2.3), the lemma is proved. 
(5.3) ThEOREM (cf. [4, (3.8)]). Let $(M, g)$ be a complete connected Riemannian manifold, $\operatorname{dim} M=2$, with $m$ eigenfunctions $f(1), \ldots, f(m), m>$ 1 , corresponding to the eigenvalue $\lambda$, which satisfy (2.1). Then either $\lambda=2 \kappa$ and $(M, g)$ is isometrically diffeomorphic to a sphere $S^{2}$ of curvature $\kappa$ or $\lambda>4 \inf _{p \in M} \kappa(p)$.

Proof. Assume $\lambda<4 \kappa$ on $M$. From (5.1) and (5.2), $\kappa$ is subharmonic on $M$; furthermore $0<\frac{1}{4} \lambda<\kappa<\frac{1}{2} \lambda$. Then $\kappa=$ const [12, p. 26]; (5.2.2), (3.4d) and (2.3) give

$$
\nabla_{k}\left(f(\alpha)_{i j}+\frac{1}{2} \lambda f(\alpha) g_{i j}\right)=0 \text { for } \alpha=1, \ldots, m .
$$

$(M, g)$ is irreducible (as $\kappa>0)$; therefore $f(\alpha)_{i j}+\frac{1}{2} \lambda f(\alpha) g_{i j}=\mu \cdot g_{i j}, \mu \in \mathbf{R}$; but $\mu=0$, as $f(\alpha)$ is an eigenfunction corresponding to $\lambda$. By Obata's theorem [6], $M$ is isometrically diffeomorphic to a sphere.

6. Minimal submanifolds of spheres. Let $\tilde{x}: M \rightarrow S^{N-1}(1)$ be an isometric minimal immersion, $N-1>n$. Denote by $j: S^{N-1}(1) \rightarrow E^{N}$ the canonical embedding such that the center of $S^{N-1}(1)$ is the origin of the canonical coordinate system of $E^{N}$. As $\operatorname{dim} M=n$ and $j \cdot \tilde{x}$ is an isometric immersion, there exist $n$ linearly independent solutions of $\Delta f+n f=0$, namely $n$ coordinate functions $x(\alpha)$, and $\Sigma_{\alpha} x^{2}(\alpha)=\langle x, x\rangle=1$, where $\langle$,$\rangle denotes the$ inner product in $E^{N}$. Let $\tilde{S}$ (resp., $S$ ) denote the squares of the lengths of the immersions $\tilde{x}$ (resp., $j \cdot \tilde{x}$ ).

We have

$$
S=\tilde{S}+n=n[n-(n-1) R], \quad \tilde{S}=n(n-1)(1-R)
$$

(cf. [8, (3)]; note that the scalar curvature in [8] differs from that in this note by the constant factor $n(n-1))$. Let

$$
x_{i j k}:=\nabla_{k} \nabla_{j} \nabla_{i} x, \quad X_{i j}:=x_{i j}+x \cdot g_{i j} ;
$$

denote now the eigenvalues of $x(\alpha)$ by $\sigma(\alpha)_{1}, \ldots, \sigma(\alpha)_{n}$; we have the following lemma (cf. [8, Lemmas 1 and 2]):

(6.2) LEMMA.

$$
\begin{aligned}
\frac{1}{2} \Delta \tilde{S} & =\sum_{\alpha} \sum_{i<j} 2 \kappa(\alpha)_{i j}\left(\sigma(\alpha)_{i}-\sigma(\alpha)_{j}\right)^{2}+\left\langle x_{i j k}, x^{i j k}\right\rangle-(n+\tilde{S}) n \\
& =\sum_{\alpha} \sum_{i<j}\left(2 \kappa(\alpha)_{i j}-1\right)\left(\sigma(\alpha)_{i}-\sigma(\alpha)_{j}\right)^{2}+\left\langle\nabla_{k} X_{i j}, \nabla^{k} X^{i j}\right\rangle .
\end{aligned}
$$

Using (3.4c), (6.1) and $1>R>\inf \kappa(p)=\kappa_{0}$, we obtain

(6.3) LeMMA. Let $\tilde{x}: M \rightarrow S^{m-1}$ (1) be minimal. (a) If $n>2$ and $\kappa_{0}>0$, then $\Delta R<2 n(1-R)\left(1-2 \kappa_{0}\right)$. (b) If $n=2$, then $\Delta \kappa<4(1-\kappa)(1-2 \kappa)$.

(6.4) Remark. As $\tilde{S}=n(n-1)(1-R),(6.3 .1)$ could be formulated as a differential inequality for $\tilde{S}$. (6.3) implies that if $\tilde{x}$ is a minimal isometric immersion with $\kappa_{0}>0$ and 


$$
\Delta R+2 n(1-R)\left(2 \kappa_{0}-1\right)>0,
$$

then $\tilde{x}$ is totally geodesic.

Lemma (6.3) implies the following result, which generalizes Theorem I in [8], where we assumed $M$ to be closed; the proof works analogously to that of Theorem (5.3) above.

(6.5) THEOREM. Let $\tilde{x}: M \rightarrow S^{m-1}(1)$ be an isometric minimal immersion, $\operatorname{dim} M>2, \kappa_{0}=\inf \kappa>0$, and let $(M, g)$ be complete. Then either $\tilde{x}(M) \subset$ $S^{m-1}(1)$ is totally geodesic or $2 \kappa_{0}<1$.

\section{REFERENCES}

1. M. Berger, P. Gauduchon and E. Mazet, Le spectre d'une variété riemannienne, Lecture Notes in Math., vol. 194, Springer-Verlag, Berlin and New York, 1971.

2. S.-Y. Cheng, A characterization of the 2-sphere by eigenfunctions, Proc. Amer. Math. Soc. 55 (1976), 379-381.

3. M. P. do Carmo and N. R. Wallach, Minimal immersions of spheres into spheres, Ann. of Math. (2) 93 (1971), 43-62.

4. F.-J. Lange, Einige Integralformeln der Riemannschen Geometrie und ihre Anwendung auf Probleme der Flächentheorie, Diplomarbeit Technische Universität Berlin, 1977.

5. S. Kobayashi and K. Nomizu, Foundations of differential geometry, vol. I. Interscience, New York, 1963.

6. M. Obata, Certain conditions for a Riemannian manifold to be isometric with a sphere, J. Math. Soc. Japan 14 (1962), 333-340.

7. U. Simon, Isometries with spheres, Math. Z. 153 (1977), 23-27.

8. Submanifolds with parallel mean curvature vector and the curvature of minimal submanifolds of spheres, Arch. Math. 29 (1977), 100-104.

9. , Curvature bounds for the spectrum of closed Einstein spaces, Canad. J. Math. 30 (1978), 1087-1091.

10. T. Takahashi, Minimal immersions of Riemannian manifolds, J. Math. Soc. Japan 18 (1966), 380-385.

11. K. Tandai, Riemannian manifolds admitting more than $(n-1)$ linearly independent solutions of $\nabla^{2} \rho+c^{2} \rho g=0$, Hokkaido Math. J. 1 (1972), 12-15.

12. K. Yano and S. Bochner, Curvature and Betti numbers, Ann. of Math. Studies, no. 32, Princeton Univ. Press, Princeton, N. J., 1953.

13. S. Tanno, On a lower bound of the second eigenvalue of the Laplacian on an Einstein space, (to appear).

Techische Universitït Berlin, Fachbereich 3-Mathematik, Strasse des 17. JUni 135, 1 Berlin 12, Federal Republic of Germany 